\title{
Cost-effectiveness and value of information analysis of a low-friction environment following skin graft in patients with burn injury
}

Rebecca Kandiyali ${ }^{1 *}$ (D), Howard Thom ${ }^{1}$, Amber E. Young ${ }^{1,2}$, Rosemary Greenwood ${ }^{2}$ and Nicky J. Welton ${ }^{1}$

\begin{abstract}
Background: Patients with burn injuries may receive a skin graft to achieve healing in a timely manner. However, in around $7 \%$ of cases, the skin graft is lost (fails to attach to the wound site) and a re-grafting procedure is necessary. It has been hypothesised that low-friction (smooth, more slippery) bedding may reduce the risk of skin-graft loss. A before and after feasibility study comparing low-friction with standard bedding in skin-grafted patients was conducted in order to collect proof of concept data. The resulting relative risk on the primary outcome (number of patients with skin graft failure) for the non-randomised study provided no evidence of effect but had a large standard error. The aim of this study is to see if an appropriately powered randomised control trial would be worthwhile.
\end{abstract}

Methods: A probabilistic decision-analytic model was constructed to compare low-friction bedding to standard care in a population of burn patients who have undergone skin grafting. Results from the before and after study were used as model inputs. The sensitivity of results to bias in the relative risk of graft loss was conducted. Low-friction bedding is considered optimal if expected incremental net benefit (INB) is positive. Uncertainty is assessed using cost-effectiveness acceptability curves. Expected Value of Perfect Partial Information (EVPPI) provides an upper bound for the potential net health benefits of new research for given model input.

Results: At a willingness to pay threshold of $£ 20,000$ per QALY, INB $=£ 151$ (95\% Credible Interval (Crl) -142 to 814), marginally favouring low-friction bedding but with high uncertainty (probability of being cost-effective 70.5\%). Expected value of perfect information (EVPI) per patient was $£ 20.29$, which results in a population EVPI of $£ 174,765$ over a 10-year lifetime for the technology (based on 1000 patients per year who would benefit from the intervention). The parameter contributing most to the uncertainty was the inpatient care cost, i.e. information that could be obtained from the audit of practice and without an expensive trial. These findings were robust to a wide-range of assumptions about the potential bias due to the observational nature of the comparative evidence.

Conclusions: Our study results suggest that an RCT (randomised controlled trial) is unlikely to be worthwhile, but there may be value in a study to estimate the re-graft rates and associated costs in this population.

Keywords: Cost-effectiveness analysis, Proof of concept study, Randomised controlled trial, Value of information analysis

\footnotetext{
* Correspondence: rebecca.kandiyali@bristol.ac.uk

'Population Health Sciences, Bristol Medical School, University of Bristol,

Bristol, UK

Full list of author information is available at the end of the article
}

(c) The Author(s). 2020 Open Access This article is distributed under the terms of the Creative Commons Attribution 4.0 International License (http://creativecommons.org/licenses/by/4.0/), which permits unrestricted use, distribution, and reproduction in any medium, provided you give appropriate credit to the original author(s) and the source, provide a link to the Creative Commons license, and indicate if changes were made. The Creative Commons Public Domain Dedication waiver (http://creativecommons.org/publicdomain/zero/1.0/) applies to the data made available in this article, unless otherwise stated. 


\section{Background}

A burn injury to the skin or other tissue occurs when cells are destroyed by hot liquids (scalds), hot solids, flames, electricity, chemicals or cold [1]. Around 130, 000 patients with burns present to English and Welsh hospitals annually; 45,000 are severe enough to require hospital follow-up [1]. Of these, 11,500 patients require admission of which $50 \%$ are children [2]. Globally, 11 million people are estimated to suffer burns significant enough to warrant medical attention [3]. Early wound closure after an injury is the aim of all modern burn care pathways resulting in improvement in survival at a substantially lower cost, better cosmetic outcomes and shorter lengths of hospital stay [4]. Skin grafts are used to treat burns to achieve healing in full-thickness burns (where the three layers of skin known as the epidermis, dermis and subcutis are damaged) or for the best cosmetic outcome in partial-thickness burns (where the top two layers, i.e. the epidermis and dermis are damaged) failing to heal within 3 weeks [5]. There are roughly 1000 skin grafts undertaken to achieve healing annually at burns services nationally; $75 \%$ in adults and $25 \%$ in children [2]. National Burns Injury database (iBID) data suggest that 20 to $30 \%$ of patients will require further grafting procedures. Some of this graft failure will be due to infection and some due to friction when the graft rubs against other materials (graft loss because of friction between bedsheets and another material, such as a bedsheet). It is difficult to determine how many of graft failures are due to friction alone. Wounds that fail to heal will cause considerable distress to patients, impacting negatively on physical, social, emotional and economic aspects of their life [6]. Graft loss will result in delayed wound healing, increased hospital stay, repeat surgery, further donor sites, increased pain and the potential for infection and increased scar formation; impacting negatively on UK National Health Service (NHS) costs.

Low-friction bedding has been shown to be a clinical and cost-effective option in the prevention of skin breakdown in a non-burns population at-risk of skin breakdown [7]. Therefore, a hypothesis is that lowfriction bedding may reduce the risk of skin-graft loss in patients with burn injury; however, the data is presently limited to non-randomised evidence from a before and after study design [8]. In brief, the Skin grafting Low friKtion Environment (SILKIE) study was a non-randomised two-centre study of the feasibility of delivering the low-friction environment with proof of concept through the comparison of retrospective with prospective data collection over 12 months. Given the low level of evidence, there remains uncertainty whether low-friction bedding prevents skin graft losses. Although low-friction bedding costs more than standard bedding, if it reduces graft loss, then this is expected to make a saving to the NHS, since re-grafting is expensive (due to the cost of the re-graft procedure and associated inpatient stay). We are therefore uncertain about both effectiveness and cost-effectiveness. This raises the question as to whether a RCT would be a worthwhile investment.

The aim of this paper is to build an economic model to assess the cost-effectiveness of low-friction bedding and use this to (i) assess uncertainty in optimal bedding given current evidence and (ii) to quantify the value of conducting a new RCT to reduce uncertainty as to the most cost-effective bedding.

The paper is organised as follows. We begin by describing the decision question and model structure. We then describe the evidence sources used to populate the model. We then present results from the cost-effectiveness analysis and value of information analysis. We finish with a discussion of the implication of the results for future research priorities.

\section{Methods}

The economic model was designed to assess costeffectiveness for the following population and interventions.

\section{Population}

Patients (adults and children) with burns who have undergone a skin graft.

\section{Intervention and comparator}

The intervention was the use of low-friction bedding in patients who are recovering from skin graft surgery. The comparator was standard bedding.

\section{Outcomes}

We calculated the Expected net benefit (ENB) and the probability of being cost-effective. ENB puts costs and QALYs onto a monetary scale using the formula: Expected Net Benefit $=$ Expected $($ QALYs) $*$ lambda +Expected (costs), where lambda represents the ceiling ratio that society is willing to pay for a gain in QALY-typically $£ 20,000$ in recommendations presided over by NICE [9]. The Expected Incremental Net Benefit is the difference between ENB on lowfriction and standard bedding, with positive values indicating that low-friction is optimal. Uncertainty in the optimal bedding is assessed by reporting credible intervals around the ENB, and also by constructing cost-effectiveness acceptability curves (CEACS) to examine the probability that the intervention is costeffective at different levels of willingness to pay (lambda). We additionally considered the expected value of perfect information (EVPI)-the expected 
Table 1 Summary of inputs used in model

\begin{tabular}{|c|c|c|c|c|}
\hline Variable & $\begin{array}{l}\text { Estimates (credible } \\
\text { interval) }\end{array}$ & Distribution & $n$ & Source/assumption \\
\hline $\begin{array}{l}\text { Relative risk (regraft) [low-friction vs. } \\
\text { standard] }\end{array}$ & $0.574(0.52,0.63)$ & $\begin{array}{l}\text { Lognormal }(-0.56 \text {, } \\
0.61)\end{array}$ & 221 & $\begin{array}{l}\text { Before and after comparison_-Silkie feasibility } \\
\text { study }\end{array}$ \\
\hline$P$ (regraft) low-friction & $0.038(0.013,0.077)$ & Beta $(4.96,125.04)$ & 90 & $\begin{array}{l}\text { Prospective data collection_-Silkie feasibility } \\
\text { study }\end{array}$ \\
\hline Intervention cost, $£$ & 115 & Deterministic & 131 & Estimate based on unit costing approach \\
\hline Cost (re-graft), $£$ & $19,321(9092,36,251)$ & $\begin{array}{l}\text { Lognormal }(9.81 \\
0.35)\end{array}$ & 131 & "l" \\
\hline Cost (no regraft), $£$ & $9908(8319,11,705)$ & $\begin{array}{l}\text { Lognormal }(9.20 \\
0.09))\end{array}$ & 131 & "'" \\
\hline Utility post surgery & $0.353(0.0056,0.91)$ & Beta $(0.77,1.41)$ & 40 & $\begin{array}{l}\text { Prospective data collection—Silkie feasibility } \\
\text { study }\end{array}$ \\
\hline Utility no graft loss & $0.508(0.11,0.90)$ & Beta $(2.26,2.19)$ & 27 & $\begin{array}{l}\text { Prospective data collection—Silkie feasibility } \\
\text { study }\end{array}$ \\
\hline Utility graft loss & $0.627(0.57,0.68)$ & Beta $(179.95,106.82)$ & 2 & \\
\hline Discount rate & $0.035(-)$ & Deterministic & $\mathrm{n} / \mathrm{a}$ & NICE methods of technology appraisal (2013) \\
\hline
\end{tabular}

value of eliminating uncertainty on all parametersand the expected value of partial perfect information (EVPPI)-the expected value of eliminating uncertainty on individual parameters. Population EVPI and EVPPI were determined by estimating the number of patients who will benefit from the technology over its lifetime.

\section{Time horizon and discounting}

Our base case model has a 28-day time horizon, informed by our clinical experts. The intervention is primarily a change to the inpatient environment and the relevant costs and outcomes relate to the period of skin graft 'take', so relative differences in costs and effects relating to the intervention are likely to occur in the short term, while the patient is hospitalised. This is in line with a previous model in a less severe burns population which had a 21-day time horizon justified on the basis of time to skin re-epithelisation and typical inpatient duration [10]. No discounting was applied to costs and benefits in the model due to the short time horizon being modelled. However, in the Population EVPI and EVPPI calculations over a 10-year lifetime horizon of the technology, an annual discount rate of $3.5 \%$ was applied [11].

\section{Model structure}

A simple probabilistic decision tree model was constructed with input from the clinical team at a tertiary burns service in Bristol, UK. In the specification of the model, patients are placed on either "Silkie" [low -friction] or standard bedding after skin graft surgery. Patients may then lose all or a sufficient proportion of their grafts such that they require a regraft; otherwise, they were not re-grafted. Figure 1 describes the structure of the 28-day decision-tree which corresponds to the decision problem which could be addressed by a full RCT.

\section{Model inputs}

Data sources used to populate the model are described in the following sections. Input parameter values and distributions used in the probabilistic sensitivity analysis are presented in Table 1.

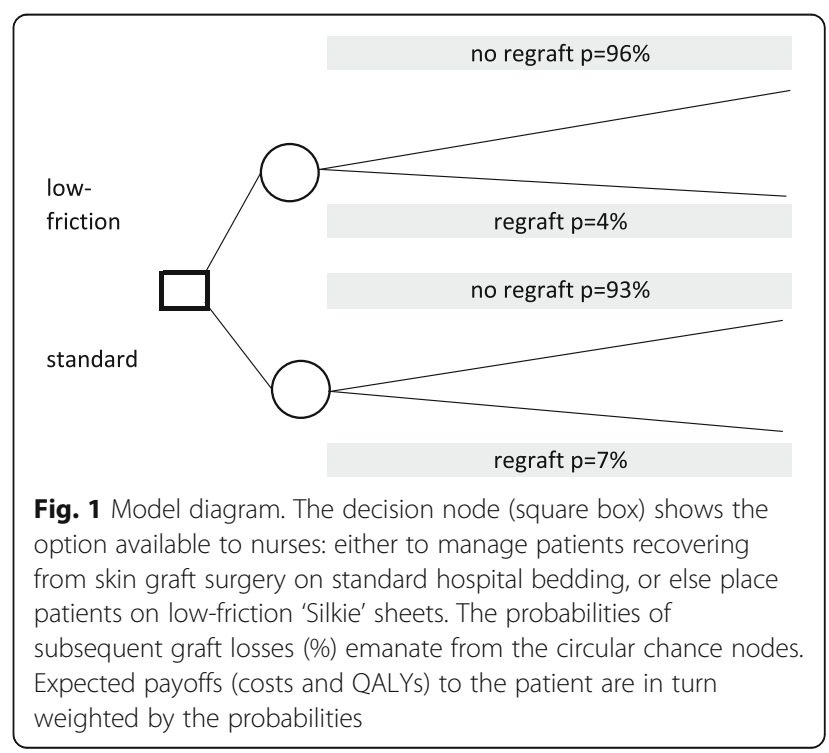




\section{Relative risk of the low-friction environment (versus standard care)}

Ideally, evidence on relative risks of re-graft would come from an RCT [12]. In the absence of this, comparative evidence from the Silkie before and after study was used. The need for re-grafting on low-friction bedding was a prospectively collected study outcome and the relative risk of low-friction versus standard was informed by the before and after comparison. Because the lack of concealed randomisation can lead to problems of comparability because of selection biases and potential confounders [13, 14] and because before/after designs are by definition prone to history bias [15], we performed multiple sensitivity analyses to assumptions on the magnitude and precision of the bias (see 'Sensitivity analyses').

\section{Absolute risk of graft loss associated with standard care} Absolute risk on standard sheets should come from representative contemporary practise. The Silkie study's before element was informed by retrospective notes review of graft losses and this informed the absolute and therefore the relative risk used in the model (as described above).

\section{Cost of re-grafting}

Ideally, the cost of re-grafting would come from microcosting studies since such studies offer the most comprehensive and rigorous methods to capture the cost associated with a specific procedure [16]. In the absence of this, we sought to use national reference costs which provide a source of unit costs based on trust activity [17]. In our analysis of inpatient costs, we multiplied the cost of an NHS reference cost 'inlier' bed-day by length of stay to estimate inpatient care costs at the level of the patient. Bed-day costs were determined by application of the appropriate reference cost category for the procedure(s). In the absence of more detailed evidence, the cost of regrafting was taken to be the incremental difference in the costs in patients who required re-grafting and those that did not, based on all patients (i.e. standard care and intervention arms) in the Silkie study.

\section{QALYs}

Ideally, data on utilities would be routinely collected in the population with burn injury to allow for service evaluation and monitoring. A populationlevel sample would greatly increase the precision around the estimate of expected QALYs. However, measurement of health-related QoL is not routine in the UK burn populations, so the utility estimates for patients who did and did not undergo re-graft surgeries were identified from the perspective (intervention arm) of Silkie study. In the first year of the model, utilities were obtained from EQ-5D utility scores recorded at baseline and 28 days follow-up (the latter stratified according to re-graft status) in the intervention arm in study participants who consented to complete questionnaires.

\section{Analysis}

The analysis was performed from the UK NHS healthcare perspective, and costs were reported in $£$ for the 2016 price year. We used a Monte Carlo simulation with 50,000 samples to propagate the joint uncertainty in model inputs into Net Benefit. In order to calculate the population level expected value of perfect information, we considered the technology relevance horizon for a technology such as this to be around 10 years. Given an estimated 1000 skin grafts per annum being carried out in England and Wales [2], expressing this in present value terms via application of a discount rate of $3.5 \%$ the expected discounted population over 10 years was 8608 . We additionally calculated the EVPPI using generalised additive regression (GAM) and integrated nested Laplace approximation (INLA) using, due to higher computational demands of GAM and INLA, 10,000 of the Monte Carlo samples [18, 19].

\section{Sensitivity analyses}

As the Silkie study used non-contemporaneous controls, it is likely the estimated relative risk and its confidence interval may be subject to bias; however, we do not know the extent of this bias. We explored the robustness of our conclusions to potential bias by presenting results for the mean bias (none, favour Silkie, favour standard), together with inflation or deflation of uncertainty (see Table 3). Details of distribution used for the relative risk of bias term are provided in Table 3.

Table 2 Costs and outcomes of providing the low-friction environment and standard care (28 days)

\begin{tabular}{llll}
\hline Intervention & Standard & Low-friction & Incremental \\
\hline Mean 28-day QALYs & $0.033(0.011,0.060)$ & $0.0332(0.010,0.060)$ & $-0.00013(-0.00073,0.00034)$ \\
Mean 28-day costs $(£)$ & $10,536(8786,12,689)$ & $10,382(8748,12,261)$ & $-154(-818,139)$ \\
Expected net benefit at $£ 20,000$ per QALY & $-9870(-12,072,-8041)$ & $-9718(-11,657,-7999)$ & $151(-142,814)$ \\
\hline
\end{tabular}




\section{Model validation}

The model was developed in $\mathrm{R}$ with the Bayesian CostEffectiveness Analysis package [20, 21]. Face validity of the model (and particularly the modelled assumptions) was established through discussion with the Silkie study management group and, health economic modellers. Internal validity was systematically checked by assessing the core calculations of the model and applying a series of logical checks to ensure that the direction of the model's predictions were consistent. We additionally used Excel to validate our calculations with respect to Net Benefit and EVPI. External and cross validity was more difficult to establish, as there were no cost-utility or value of information studies in the target population with which to compare our results.

\section{Reporting}

We used the CHEERS reporting guidelines for Economic Evaluation [22].

\section{Results}

Table 2 presents the results from the probabilistic model over 28 days for the low-friction and standard bedding. The probabilistic analyses incorporate the uncertainty around the point estimates of input parameters (Fig. 2). In the base-case, where the Silkie study results are taken at face value, the expected Incremental Net Benefit was $£ 151$ in favour of low-friction bedding at a threshold of $£ 20,000$ per QALY gained. However, there was considerable uncertainty around this estimate (95\% CrI $£-142$ to 814) suggesting that there is no clear optimal strategy based on ENB. Figure 3 presents the cost-

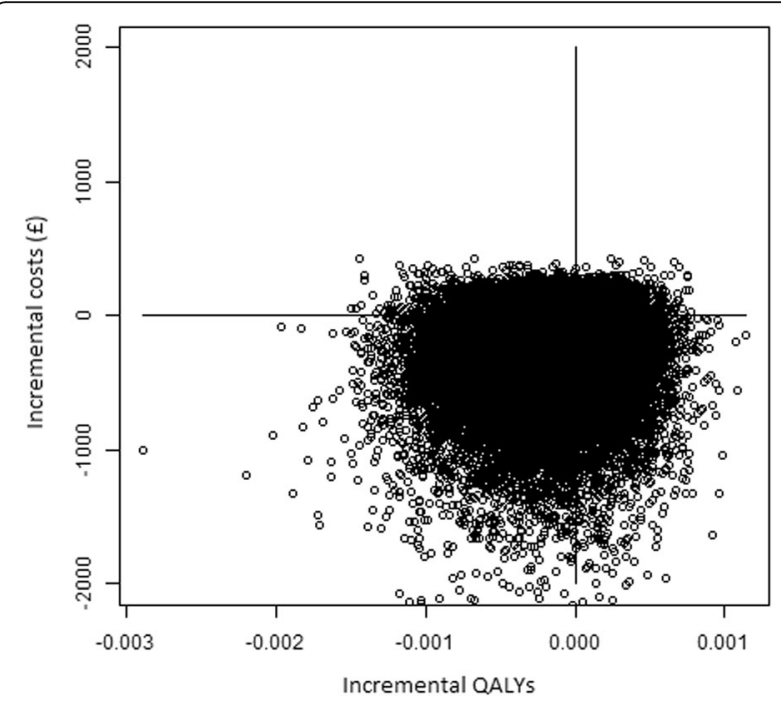

Fig. 2 Scatterplot of cost and effect pairs for low-friction bedding compared to standard care (28 days)

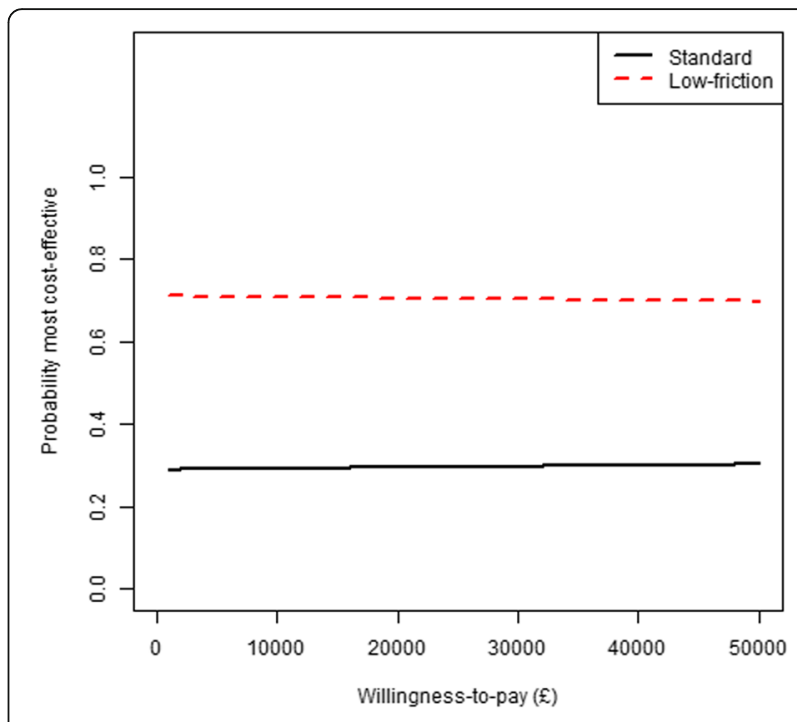

Fig. 3 Cost-effectiveness acceptability curve

effectiveness acceptability curves which shows that low-friction bedding has a $70.5 \%$ chance of being cost-effective at a threshold of $£ 20,000$ per QALY gained. Table 3 shows the Population EVPI over the 28-day time horizon is $£ 20.29$ per person at a threshold value of $£ 20,000$. For a decision relevance horizon of 10 years, this corresponds to an overall expected value of removing decision uncertainty for England and Wales of $£ 174,675$.

Figure 4 describes the EVPPI for individual parameters. This shows that the parameters causing most of the decision uncertainty are around costs and the absolute probability for re-grafting surgery, regardless of the sheeting environment. Table 3 also shows the results of the sensitivity analysis for bias in the relative risk, and implications in terms of expected net-benefit, the probability that low-friction is most cost-effective, EVPI and EVPPI parameters.

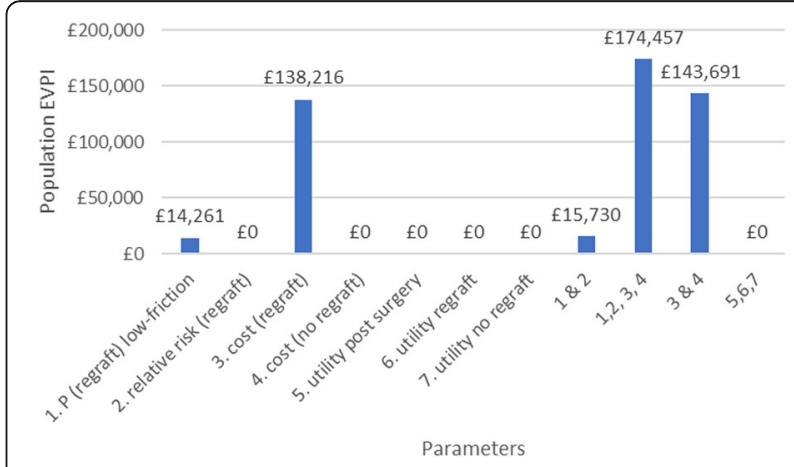

Fig. 4 Expected value of perfect information in individual parameters 


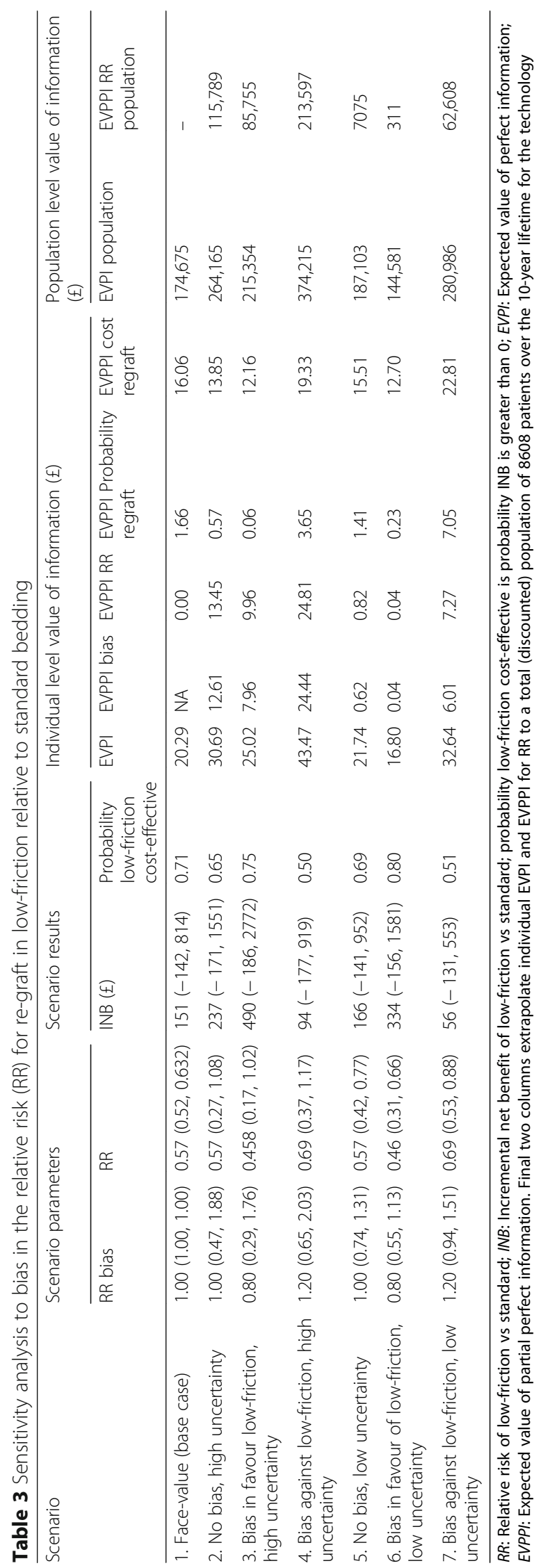


EVPPI for further parameter sets under bias scenarios is provided in the Appendix. The probability that low-friction is most cost-effective is higher when bias is upwards (favouring low-friction) and when bias is least uncertain. We see that the EVPI is greatest when the benefit of low-friction vs standard is most uncertain (bias against low-friction bedding with high uncertainty, scenario 4) and lowest when the benefit is most certain (bias in favour of low-friction and low uncertainty, scenario 6). The parameters with largest EVPPI, and with EVPPI that changes most under bias scenarios, are the probability of re-graft, relative risk of re-graft, and the cost of re-graft; utilities appear to have little influence on decision making and are unaffected by assumptions about bias in the relative risk (see Appendix). The final column represents the value of an RCT exploring the relative risk, with no value in the base case but the highest value if we assume the observational evidence is biased against lowfriction bedding and highly uncertain (scenario 4) or unbiased but highly uncertain (scenario 2). Regardless of the assumptions about bias, there does not seem to be value in an RCT of low-friction vs standard bedding. However, there may be value in conducting another type of study to collect re-graft costs as population EVPPI for this parameter was $£ 138,216$. This would be much cheaper and probably worthwhile (given the data would be relevant for other decision questions for such patients in the future).

\section{Discussion}

This paper reports on the construction of a model to evaluate the cost-effectiveness of a low-friction environment in patients who have had recent skin graft surgery, and the potential value of future research. Our results suggest that there is no value in a future RCT regardless of how large we believe the bias from the before-after Silkie study to be because the cost of an RCT would far outweigh the benefits from it as measured by EVPPI. However, we cannot be sure which is the optimal treatment. This suggests that the consequences of using low-friction or standard bedding are not sufficiently high for us to be concerned if the wrong choice is made. However, we found that there may be value in studies collecting data on the cost of re-graft surgery and the absolute probability of re-graft (regardless of bedding type). These data could be used to update the model and revisit the decision on bedding, and also would be valuable to inform decisions on other interventions for this population in the future.

\section{Summary of findings elsewhere}

We found no published evaluations in burn management using cost-effectiveness modelling. Brown, David et al. [23] published an economic evaluation of a distraction-based intervention 'Ditto' in paediatric burn care in Brisbane, Australia based on a small trial $(n=$ 75). The study reported a $95 \%$ probability that it was cost-effective compared with standard care, but the results are not easily comparable since they report cost (AU\$) per 1-day reduction in re-epithelialization. Elsewhere, Tuffaha et al. identify the EVPI for the decision to adopt negative pressure wound therapy at AUD 2.7 million, which places a much greater value on more research than for our study [24]. We found evidence of an unpublished UK economic evaluation for a spray-on skin system in patients with burn injury [10] but the model developed was only applicable to patients with partial burns that do not require re-grafting due to a lack of evidence in grafted patients and did not consider value of information. Additionally, we are aware of modest literature on the costs associated with burn injury in child and adult populations. However, these studies are typically either very small or report costs based on different healthcare systems so may not be generalisable [25-30].

\section{Limitations}

One limitation is that we present modelled results drawing on a retrospective comparison of patients nursed on standard bedding, in a preceding 12-month period in the same hospitals. This lack of a contemporaneous and prospectively collected comparison group is a key limitation of inference on the basis of feasibility study results. While we explored these in scenario analyses the nature of bias (some which may be unobservable) is that we do not know if the analyses we conducted are reasonable and explored the full range of possibilities.

We note that the short-time horizon of this model made it unlikely that we would identify differences in QALYs, and this may mean that we did not consider the inclusion of potentially relevant effects. These effects could be substantial if repeat skin grafts cause long-term or permanent scarring and the utility decrement (and the associated loss in QALYs) can be quantified. Further work could set out to collect data on utilities or elicit informative priors to describe uncertainty in these parameters [31] in order to populate a long-term model. Any future model development should foreseeably involve patients and the public to validate such long-term assumptions.

\section{Conclusion}

Our model for the UK population suggests that a further definitive trial is not worthwhile and instead low-cost research audits should focus on the re-graft rates and cost of graft surgeries. 


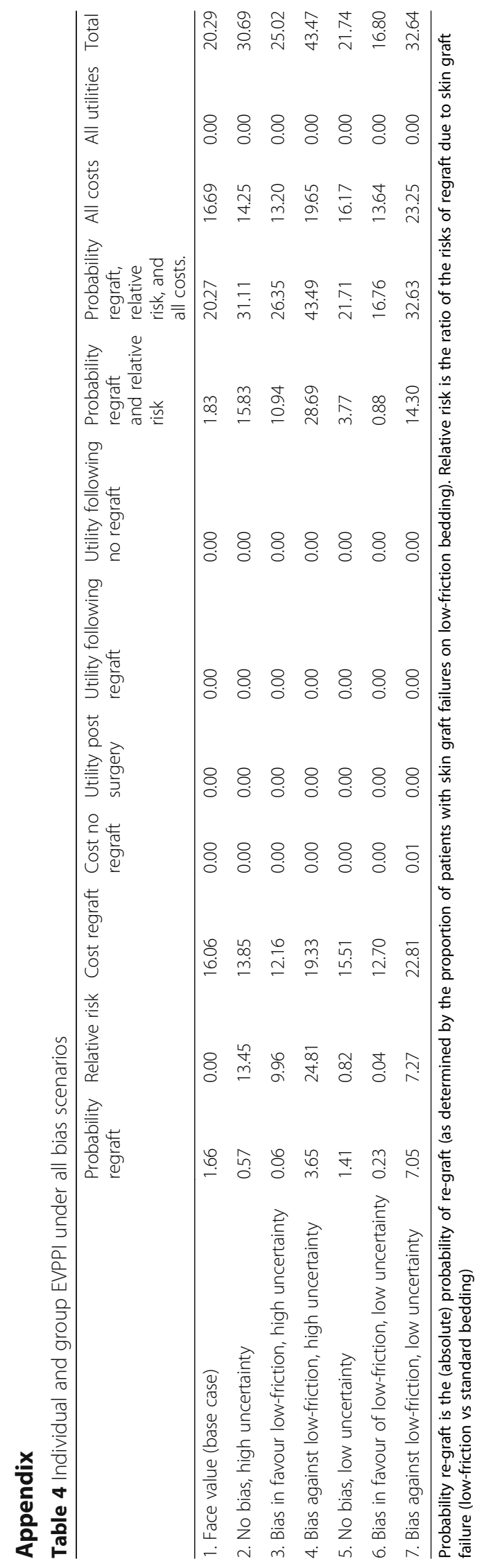




\section{Abbreviations}

Crl: Credible interval; ENB: Expected net benefit; EVPI: Expected value of perfect information; EVPPI: Expected value of perfect partial information; iBID: National Burns Injury Database; NHS: National Health Service; QALY: Quality-adjusted life year; RCT: Randomised controlled trial

\section{Acknowledgements}

RK wishes to thank Health Economics at Bristol for the consideration of an earlier version of this paper at its in-house research forum.

\section{Authors' contributions}

RK conceived of the idea, developed the model structure, identified data inputs and drafted the paper. HT wrote the code for the model in R and conducted the analysis. NW guided on the interpretation of the model and inclusion of a bias analysis. RG and AEY provided expert input with respect to the use of trial data from the Silkie study. All authors contributed to and approved the final manuscript.

\section{Funding}

While this study was not directly funded, RK and AEY received funding from the Scar Free Foundation Centre for Children's Burn Research between 2015 and 18. Additionally, the Silkie study was supported by the National Institute for Health Research (NIHR) under its Research for Patient Benefit (RfPB) Programme (Grant Reference No. PB-PG-0214-33003). HT and NW received support from the UK Medical Research Council ConDuCT-II Hub for Trials Methodology Research (MR/K025643/1) and from the NIHR Biomedical Research Centre at University Hospitals Bristol NHS Foundation Trust and the University of Bristol.

\section{Availability of data and materials}

The $\mathrm{R}$ code for the model is available from the corresponding author on reasonable request.

\section{Ethics approval and consent to participate}

This model is secondary research, however, the before and after study which provided the absolute and relative risks for the model had the ethical approval from The National Institute for Social Care and Health Research Ethics Service Committee Wales REC 4 (REC reference: 15/WA/0156)

\section{Consent for publication}

Not applicable.

\section{Competing interests}

The authors declare no competing interests. HT reports personal fees for consultancy work from Pfizer, Novartis Pharma, Hoffman La-Roche, Bayer, Janssen, and Eli Lilly and company, all outside this work.

\section{Author details}

${ }^{1}$ Population Health Sciences, Bristol Medical School, University of Bristol,

Bristol, UK. 'University Hospitals Bristol NHS Foundation Trust, Bristol, UK.

Received: 5 June 2019 Accepted: 17 December 2019

Received: 5 June 2019 Accepted: 17 December

\section{References}

1. Kagan RJ, Peck MD, Ahrenholz DH, Hickerson WL, Holmes J, Korentager R, et al. Surgical management of the burn wound and use of skin substitutes: an expert panel white paper. J Burn Care Res. 2013;34(2):e60-79.

2. Dunn K. iBID database report: numbers and burn types in children under 5 years of age (09.2007 to 08.2012); 2013.

3. Stokes MAR, Johnson WD. Burns in the third world: an unmet need. Ann Burns Fire Disasters. 2017;30(4):243-6.

4. Atiyeh BS, Hayek SN, Gunn SW. New technologies for burn wound closure and healing--review of the literature. Burns. 2005;31(8):944-56.

5. Cubison TC, Pape SA, Parkhouse N. Evidence for the link between healing time and the development of hypertrophic scars (HTS) in paediatric burns due to scald injury. Burns. 2006;32(8):992-9.

6. Andersson AE, Bergh I, Karlsson J, Nilsson K. Patients' experiences of acquiring a deep surgical site infection: an interview study. Am J Infect Control. 2010;38(9):711-7.
7. Smith $G$, Ingram A. Clinical and cost effectiveness evaluation of low friction and shear garments. J Wound Care. 2010;19(12):535-42.

8. Hollén L, Greenwood R, Kandiyali R, Ingram J, Foy C, George S, et al. The SILKIE (Skin graftIng Low friKtlon Environment) study: a nonrandomised proof-of-concept and feasibility study on the impact of low-friction nursing environment on skin grafting success rates in adult and paediatric burns. BMJ Open. 2018;8(6):e021886.

9. Briggs AH, Claxton K, Sculpher MJ. Decision modelling for health economic evaluation. Oxford: Oxford University Press; 2006.

10. NICE. The ReCell ${ }^{\oplus}$ Spray-On Skin ${ }^{\oplus}$ system for treating skin loss, scarring and depigmentation after burn injury: Medical Technologies Evaluation Programme; 2014 Available from: https:/mww.nice.org.uk/guidance/mtg21. Accessed 26 Jan 2017

11. NICE. Guide to the methods of technology appraisal. 2013.

12. Murad MH, Asi N, Alsawas M, Alahdab F. New evidence pyramid. Evid Based Med. 2016:21(4):125-7.

13. Higgins JPT, Green S, editors. Cochrane handbook for systematic reviews of interventions version 5.1.0 [updated March 2011]: The Cochrane Collaboration; 2011. Available from www.handbook.cochrane.org

14. Goodacre S. Uncontrolled before-after studies: discouraged by Cochrane and the EMJ. Emerg Med J. 2015;32(7):507-8.

15. Ho AMH, Phelan R, Mizubuti GB, Murdoch JAC, Wickett S, Ho AK, et al. Bias in before-after studies: narrative overview for anesthesiologists. Anesth Analg. 2018;126(5):1755-62.

16. Xu X, Grossetta Nardini HK, Ruger JP. Micro-costing studies in the health and medical literature: protocol for a systematic review. Syst Rev. 2014;3:47.

17. Department of Health. National reference costs 2014-15. Available from https://www.gov.uk/government/uploads/system/uploads/attachment_ data/file/477919/2014-15_Reference_costs_publication.pdf. 2015.

18. Heath A, Manolopoulou I, Baio G. Estimating the expected value of partial perfect information in health economic evaluations using integrated nested Laplace approximation. Stat Med. 2016;35(23):4264-80.

19. Strong M, Oakley JE, Brennan A. Estimating multiparameter partial expected value of perfect information from a probabilistic sensitivity analysis sample: a nonparametric regression approach. Med Decis Mak. 2014;34(3):311-26.

20. R: a language and environment for statistical computing. 2018. Available from: https://www.R-project.org. Accessed 7 May 2019.

21. Baio $G$, Berardi A, Heath A. Bayesian cost-effectiveness analysis with the $R$ package BCEA, in use R! Springer International Publishing; 2017.

22. Husereau D, Drummond M, Petrou S, Carswell C, Moher D, Greenberg D, et al. Consolidated health economic evaluation reporting standards (CHEERS) statement. BMJ. 2013:346:f1049.

23. Brown NJ, David M, Cuttle L, Kimble RM, Rodger S, Higashi H. Costeffectiveness of a nonpharmacological intervention in pediatric burn care. Value Health. 2015;18(5):631-7.

24. Tuffaha HW, Gillespie BM, Chaboyer W, Gordon LG, Scuffham PA. Cost-utility analysis of negative pressure wound therapy in high-risk cesarean section wounds. J Surg Res. 2015;195(2):612-22.

25. Kandiyali R, Sarginson JH, Hollen LI, Spickett-Jones F, Young AER. The management of small area burns and unexpected illness after burn in children under five years of age - A costing study in the English healthcare setting. Burns: journal of the International Society for Burn Injuries. 2018;44(1):188-94.

26. Klein MB, Hollingworth W, Rivara FP, Kramer CB, Askay SW, Heimbach DM, et al. Hospital costs associated with pediatric burn injury. J Burn Care Res. 2008:29(4):632-7.

27. Eser T, Kavalci C, Aydogan C, Kayipmaz AE. Epidemiological and cost analysis of burn injuries admitted to the emergency department of a tertiary burn center. Springerplus. 2016;5(1):1411

28. Hop MJ, Polinder S, Middelkoop E, van Baar ME. Costs of burn care: a systematic review. Value Health. 2014;17(7):A606.

29. Carey K, Kazis LE, Lee AF, Liang MH, Li NC, Hinson Ml, et al. Measuring the cost of care for children with acute burn injury. J Trauma Acute Care Surg 2012:73(3 Suppl 2):S229-33.

30. Griffiths HR, Thornton KL, Clements CM, Burge TS, Kay AR, Young AE. The cost of a hot drink scald. Burns. 2006:32(3):372-4.

31. Soares MO, Bojke L, Dumville J, Iglesias C, Cullum N, Claxton K. Methods to elicit experts' beliefs over uncertain quantities: application to a cost effectiveness transition model of negative pressure wound therapy for severe pressure ulceration. Stat Med. 2011;30(19):2363-80.

\section{Publisher's Note}

Springer Nature remains neutral with regard to jurisdictional claims in published maps and institutional affiliations. 